\title{
Quantum dynamics of a damped deformed oscillator
}

\author{
Stefano Mancini \\ Dipartimento di Fisica and Unità INFM, Università di Milano, \\ Via Celoria 16, I-20133 Milano, Italy
}

\begin{abstract}
The interaction of a quantum deformed oscillator with the environment is studied deriving a master equation whose form strongly depends on the type of deformation.
\end{abstract}

PACS number(s): 03.70.+k, 02.50.-r

Quantum groups [1] introduced as a mathematical description of deformed Lie algebras have given the possibility to generalize the notion of creation and annihiliation operators of the usual oscillator and to introduce $q$-oscillators [2, 3]. Soon after, several attempts have been made to give a physical interpretation of the $q$-oscillators. In Ref. [4] they appear in connection with the relativistic oscillator model, while in Ref. [5] $q$-oscillators have been used in the generalized Jaynes-Cummings model.

Instead, the nature of $q$-oscillators of the electromagnetic field is clarified by the nonlinearity of the field vibrations [6]. This $q$-nonlinearity reflects the exponential growth of the frequency of vibrations with the amplitude.

This observation suggests that there might exist other types of nonlinearities for which the frequency of oscillation varies with the amplitude via a generic function $f$; this leads to the concept of $f$-deformed oscillators [7]. Then, the generation of coherent states for a class of $f$-deformed oscillators enter in the real possibilities of trapped systems $[8]$.

By virtue of possible further physical realizations of $f$-oscillators, it could be interesting to study the environmental effects on such oscillators.

The aim of this paper is to describe the behavior of a nonlinear oscillator, like the $f$-oscillator, plunged in a bath modeled by an assembly of harmonic oscillators. A master equation approach is developed to consider several types of reservoir, restricted however to the case of small damping.

The free Hamiltonian of an $f$-deformed oscillator may be taken, in the case of unitary frequency and natural units, as [7]

$$
\mathcal{H}_{0}=\frac{1}{2}\left(A^{\dagger} A+A A^{\dagger}\right)
$$


where the operators $A$ and $A^{\dagger}$ result as a distortion of the usual annihiliation and creation operators $a$ and $a^{\dagger}$,

$$
\begin{aligned}
A & =a f(n)=f(n+1) a, \quad n=a^{\dagger} a, \\
A^{\dagger} & =f(n) a^{\dagger}=a^{\dagger} f(n+1) .
\end{aligned}
$$

Here, $f$ is intended as an operator-valued function of the number operator (moreover it is assumed Hermitian). In general, it can be made dependent on continous parameters, in such a way that, for given particular values, the usual algebra is reconstructed. This is the case of $q$-deformations [9]. As a consequence of the noncanonical transformations (2) and (3), the commutation relation is not preserved,

$$
\left[A, A^{\dagger}\right]=(n+1) f^{2}(n+1)-n f^{2}(n) .
$$

Of course the usual algebra is restored whenever $f \rightarrow 1$. Furthermore, the Eq. (1) can be rewritten as

$$
\mathcal{H}_{0}=\frac{1}{2}\left[n f^{2}(n)+(n+1) f^{2}(n+1)\right],
$$

from which results more clear its nonlinear character.

Now, the usual model of reservoir consisting in a large number of harmonic oscillators is employed by means of the Hamiltonian $\mathcal{H}_{B}=\sum_{j} \omega_{j} b_{j}^{\dagger} b_{j}$ and the linear interaction between system and bath [10]

$$
\mathcal{H}_{\text {int }}=a \Gamma^{\dagger}+a^{\dagger} \Gamma \text {. }
$$

The bath operator $\Gamma$ is given by $\Gamma=\sum_{j} \kappa_{j} b_{j}$, with $\kappa_{j}$ the frequency dependent coupling constants. In write down Eq. (6), we have assumed that the frequency of bath modes most strongly interacting with the system is very large compared to the strength of the interaction (measured in frequency units). This assumption is also known as Rotating Wave Approximation [10].

We imagine that the interaction (6) is switched on at the time $t=0$ and the bath at this time have no correlations with the oscillator. The initial joint density operator $w(0)$ can thus be taken as the product of some unspecified operator $\rho(0)$ and a given operator $\rho_{B}(0)$ for the bath. It obeys the Liouville equation

$$
\dot{w}(t)=-i\left[\mathcal{H}_{0}+\mathcal{H}_{B}+\mathcal{H}_{\text {int }}, w(t)\right] \equiv L w(t),
$$

where the superoperator $L$ should be intended as the sum $L_{0}+L_{B}+L_{\text {int }}$. The reduced density operator of the oscillator will be

$$
\rho(t)=\operatorname{Tr}_{B}\left\{e^{L t} w(0)\right\} \equiv U(t) \rho(0)
$$

Assuming the inverse of the operator $U(t)$ to exist, we may write $\rho(0)=U(t)^{-1} \rho(t)$, and thus the formal equation of motion

$$
\dot{\rho}(t)=\dot{U}(t) U(t)^{-1} \rho(t) \equiv \mathcal{L}(t) \rho(t) .
$$

Restricting ourselves to the weak damping case, $\mathcal{L}(t)$ can be constructed perturbatively by an expansion in powers of the interaction part $L_{\text {int }}$ of the Liouvillian superoperator 
$L$. Hence, neglecting the third and higher order terms, the reduced equation of motion is obtained

$$
\begin{aligned}
& \dot{\rho}(t)=L_{0} \rho(t)+\int_{0}^{t} d t^{\prime} \quad\left\{\left\langle\Gamma\left(t^{\prime}\right) \Gamma^{\dagger}\right\rangle\left[a\left(-t^{\prime}\right) \rho(t), a^{\dagger}\right]+\left\langle\Gamma^{\dagger} \Gamma\left(t^{\prime}\right)\right\rangle\left[a^{\dagger}, \rho(t) a\left(-t^{\prime}\right)\right]\right. \\
& +\left\langle\Gamma\left(t^{\prime}\right) \Gamma\right\rangle\left[a^{\dagger} \rho(t), a^{\dagger}\left(-t^{\prime}\right)\right]+\left\langle\Gamma^{\dagger} \Gamma^{\dagger}\left(t^{\prime}\right)\right\rangle\left[a \rho(t), a\left(-t^{\prime}\right)\right] \\
& + \text { H.c. }\} \text {, }
\end{aligned}
$$

where the expectation values are taken on the bath and represent its correlation functions.

To go on, it is also necessary the explicit time dependence of the operators $a$ and $a^{\dagger}$; from Eq. (5), it is

$$
a(t)=\exp [-i \Omega(n) t] a
$$

with

$$
\Omega(n)=\frac{1}{2}\left[(n+2) f^{2}(n+2)-n f^{2}(n)\right],
$$

an operator-valued frequency constant in time.

In Eq. (10), by assuming a short correlation time of the bath operators (shorter than any typical system periods), the extreme of integration $t$ can be replaced by $\infty$. Furthermore, it is possible to invoke the fluctuation-dissipation theorem in the following form [10]

$$
\begin{aligned}
\int_{-\infty}^{+\infty} d t\left\langle\Gamma(t) \Gamma^{\dagger}\right\rangle e^{i \Omega t} & =\chi(\Omega)[1+N(\Omega)] \\
\int_{-\infty}^{+\infty} d t\left\langle\Gamma^{\dagger} \Gamma(t)\right\rangle e^{i \Omega t} & =\chi(\Omega) N(\Omega) \\
\int_{-\infty}^{+\infty} d t\langle\Gamma \Gamma(t)\rangle e^{i \Omega t} & =\chi(\Omega) M(\Omega)
\end{aligned}
$$

where

$$
\chi(\Omega)=g(\Omega) \kappa^{2}(\Omega),
$$

is the bath response function determined by the density function $g$ of the bath modes in the continuum limit [10]. Hence, the master equation (10) becomes

$$
\begin{aligned}
\dot{\rho}(t) & =-i\left[\mathcal{H}_{0}, \rho(t)\right] \\
& +\frac{i}{2 \pi} P . V . \int d \Omega^{\prime} \chi\left(\Omega^{\prime}\right)\left\{\left(1+N\left(\Omega^{\prime}\right)\right)\left[\left(\Omega-\Omega^{\prime}\right)^{-1} a \rho(t), a^{\dagger}\right]\right. \\
& +N\left(\Omega^{\prime}\right)\left[a^{\dagger}, \rho(t)\left(\Omega-\Omega^{\prime}\right)^{-1} a\right]+M\left(\Omega^{\prime}\right)\left[a^{\dagger} \rho(t), a^{\dagger}\left(\Omega-\Omega^{\prime}\right)^{-1}\right] \\
& \left.+M^{\dagger}\left(\Omega^{\prime}\right)\left[a \rho(t),\left(\Omega-\Omega^{\prime}\right)^{-1} a\right]-\text { H.c. }\right\} \\
& +\frac{1}{2}\left\{\left[(1+N(\Omega)) \chi(\Omega) a \rho(t), a^{\dagger}\right]+\left[a^{\dagger}, \rho(t) N(\Omega) \chi(\Omega) a\right]\right. \\
& \left.+\left[a^{\dagger} \rho(t), a^{\dagger} M(\Omega) \chi(\Omega)\right]+\left[a \rho(t), M^{\dagger}(\Omega) \chi(\Omega) a\right]+\text { H.c. }\right\},
\end{aligned}
$$


where P.V. denotes the Cauchy principal value and the following relation has been used

$$
\int_{0}^{\infty} d t e^{i \Omega t} \mathcal{F}(t)=\frac{1}{2} \tilde{\mathcal{F}}(\Omega)+\frac{i}{2 \pi} P . V . \int_{-\infty}^{+\infty} d \Omega^{\prime}\left(\Omega-\Omega^{\prime}\right)^{-1} \tilde{\mathcal{F}}\left(\Omega^{\prime}\right),
$$

with $\mathcal{F}$ a generic bath correlation function and $\tilde{\mathcal{F}}$ its Fourier transform.

Now, the Markov approximation $\chi(\Omega)=2 \gamma$ [10], can be introduced in the second term with curly brackets in Eq. (17), while it is inadmissible in the terms involving the principal value frequency integrals since they become divergent. The latter are the analogous of the Stark and Lamb shifts in the common theory of damping [10]. To simplify the treatment, however, these terms are disregarded from now on, due to their small contribution.

A suitable basis to represent the master equation (17) in $c$-number form is obviously the number states basis, then one gets

$$
\begin{aligned}
\dot{\rho}_{m, n}(t) & =-\frac{i}{2}\left[m f^{2}(m)+(m+1) f^{2}(m+1)-n f^{2}(n)-(n+1) f^{2}(n+1)\right] \rho_{m, n}(t) \\
& -\gamma\{m[1+N(\Omega(m-1))]+n[1+N(\Omega(n-1))] \\
& +(m+1) N(\Omega(m))+(n+1) N(\Omega(n))\} \rho_{m, n}(t) \\
& +\gamma \sqrt{(m+1)(n+1)}[2+N(\Omega(m))+N(\Omega(n))] \rho_{m+1, n+1}(t) \\
& +\gamma \sqrt{m n}[N(\Omega(m-1))+N(\Omega(n-1))] \rho_{m-1, n-1}(t) \\
& +\gamma \sqrt{m(n+1)}[M(\Omega(n))+M(\Omega(m-1))] \rho_{m-1, n+1}(t) \\
& +\gamma \sqrt{(m+1) n}\left[M^{*}(\Omega(m))+M^{*}(\Omega(n-1))\right] \rho_{m+1, n-1}(t) \\
& -\gamma \sqrt{m(m-1)} M(\Omega(m-1)) \rho_{m-2, n}(t) \\
& -\gamma \sqrt{n(n-1)} M^{*}(\Omega(n-1)) \rho_{m, n-2}(t) \\
& -\gamma \sqrt{(m+1)(m+2)} M^{*}(\Omega(m)) \rho_{m+2, n}(t) \\
& -\gamma \sqrt{(n+1)(n+2)} M(\Omega(n)) \rho_{m, n+2}(t)
\end{aligned}
$$

with $\rho_{m, n}(t) \equiv\langle m|\rho(t)| n\rangle$.

It is worth noting how the system deformation strongly affects the structure of the master equation (19) also in its irreversible terms through $\Omega$. Instead, usually the nonlinearities are included in the master equation only through the term $L_{0}$, so that the damping terms remain unaffcted by the nonlinearities. This seems incorrect, unless $M(\Omega)$ and $N(\Omega)$ go to zero.

A further simplification is made by assuming to have only a thermal bath, so that

$$
M(\Omega)=0 ; \quad N(\Omega)=\frac{1}{\exp (\beta \Omega)-1},
$$

where $\beta=1 / k_{B} T ; k_{B}$ is the Boltzmann constant and $T$ the temperature. In such a case the stationary solution of the Eq. (19) can be easily found

$$
\rho_{m, n}(\infty)=\delta_{m, n} \mathcal{Z}^{-1} \exp \left[-\frac{\beta}{2}\left(n f^{2}(n)+(n+1) f^{2}(n+1)\right)\right],
$$


where $\mathcal{Z}$ is a normalization constant. The solution (21) may result far from a trivial Boltzmann distribution when a deformation is present; for example, in the case of a (small) $q$-type nonlinearity it immediately gives the deformed Planck distribution derived in Ref. [11].

Finally, it is to remark that the derived master equation is not of the Lindblad form [12], and this inevitably leads to situations in which unphysical density operator will arise (one illuminating example could be given by the harmonious deformation [13], for which $f(n)=1 / \sqrt{n})$. Nevertheless, this is not a reason to abandon this equation, it only means that one has to be careful in applying it, evaluating the range of validity for the kind of considered deformation.

Summarizing, a quite general master equation describing the damped dynamics of a deformed oscillator has been proposed, showing how its structure reflects the nonlinear character of the oscillator. The specific solution strongly depends on the type of introduced deformation. On the other hand, it could be also searched by transforming the master equation in a partial differential equation for a quasiprobability distribution [14], exploring possible connection among deformation parameter(s) and ordering parameter(s) [14].

\section{Acknowledgments}

I express my gratitude to V. I. Man'ko for introducing me to the subject of deformed algebras.

\section{References}

[1] V. G. Drinfeld, Proc. Int. Congress of Mathematicians, 1, 798 (1986); M. Jimbo, Lett. Math. Phys. 11, 247 (1986).

[2] L. C. Biedenharn, J. Phys. A 22, L873 (1989).

[3] A. J. Macfarlane, J. Phys. A 22, 4581 (1989).

[4] R. M. Mir-Kasimov, in Lecture Notes in Physics, Vol. 382. Proc. 18th Colloquium on Group Theoretical Methods in Physics, (Springer, Berlin, 1991), p.215.

[5] M. Chaichian, D. Ellinas and P. Kulish, Phys. Rev. Lett. 65, 980 (1990).

[6] V. I. Man'ko, G. Marmo, S. Solimeno and F. Zaccaria, Int. J. Mod. Phys. A 8, 3577 (1993).

[7] V. I. Man'ko, G. Marmo, F. Zaccaria and E. C. G. Sudarshan, Proc. 4th Wigner Symposium, N. Atakishiyev, T. Seligman and K. B. Wolf Eds. (World Scientific, Singapore, 1996), p.421; Physica Scripta, 55, 528 (1997).

[8] R. L. de Matos Filho and W. Vogel, Phys. Rev. A, 54, 4560 (1996).

[9] A. P. Ploychronakos, Mod. Phys. Lett. A 5, 2335 (1990).

[10] see e.g., W. Louisell, Quantum Statistical Properties of Radiation, (Wiley, New York, 1989). 
[11] V. I. Man'ko, G. Marmo, S. Solimeno and F. Zaccaria, Phys. Lett. A 176, 173 (1993).

[12] G. Lindblad, Commun. Math. Phys. 48, 119 (1976).

[13] E. C. G. Sudarshan, Int. J. Theor. Phys. 32, 1069 (1993).

[14] K. E. Cahill and R. J. Glauber, Phys. Rev. 177, 1882 (1969). 